DOI: 10.2478/awutp-2018-0011

\title{
ONLINE FORECASTING OF THE SOLAR ENERGY PRODUCTION
}

\author{
Marius Paulescu ${ }^{1}$, Nicoleta Stefu ${ }^{1}$, Ciprian Dughir ${ }^{2}$, Robert Blaga ${ }^{1}$, Andreea Sabadus ${ }^{1}$, \\ Eugenia Paulescu ${ }^{1}$, Sorin Bojin ${ }^{1}$ \\ ${ }^{I}$ Faculty of Physics, West University of Timisoara, V. Parvan Ave.4, 300223 Timisoara, Romania \\ ${ }^{2}$ Electronics and Telecommunications Faculty, Politehnica University Timisoara, V Parvan 2, 300223
}

Timisoara, Romania

\begin{tabular}{|c|c|}
\hline Article Info & Abstract \\
\hline $\begin{array}{l}\text { Received: } 21.08 .2018 \\
\text { Accepted: } 22.08 .2018 \\
\text { Keywords: solar } \\
\text { irradiance, PV power, } \\
\text { forecasting. }\end{array}$ & $\begin{array}{l}\text { Forecasting the solar energy production is a key issue in the large-scale } \\
\text { integration of the photovoltaic plants into the existing electricity grid. This paper } \\
\text { reports on the research progress in forecasting the solar energy production at the } \\
\text { West University of Timisoara, Romania. Firstly, the experimental facilities } \\
\text { commissioned on the Solar Platform for testing the forecasting models are briefly } \\
\text { described. Secondly, a new tool for the online forecasting of the solar energy } \\
\text { production is introduced. Preliminary tests show that the implemented procedure is } \\
\text { a successful trade-off between simplicity and accuracy. }\end{array}$ \\
\hline
\end{tabular}

\section{Introduction}

The power injected into the electricity grid by a photovoltaic (PV) plant depends essentially on the weather conditions. Since the response time of a PV plant is very small, the output power of the PV plant closely follows the variation of solar irradiance. Often, the deterministic variation of the solar irradiance (due to Earth's movements) is accompanied by fluctuations having an erratic character (generated by passing clouds) [1]. The sudden variation of the solar irradiance can generate the so-called solar ramp event, a term that refers to the grid management when the solar irradiance experiences fast changes, causing equally jumps in the PV output power (see e.g. [2] for a helpful introduction to solar ramp events). It is well documented that the accurate forecasting of the output power of a PV plant allows for an efficient grid management and increases its operational safety [3]. The accuracy of forecasting the output power of a PV plant is largely determined by the accuracy of forecasting the solar irradiance. In the last years, a huge effort has been paid around the world 
to increase the forecasts accuracy [4, 5]. Our research group (http://solar.physics.uvt.ro) has been constantly involved in this general effort since $2010[6,7]$. This paper reports on our results in developing a toolkit for forecasting the energy production of a PV plant. The paper is organized as follows. The experimental setup is briefly described in Sec. 2. The relevant data used in this study are presented in Sec. 3. The online forecasting procedure is introduced in Sec. 4.

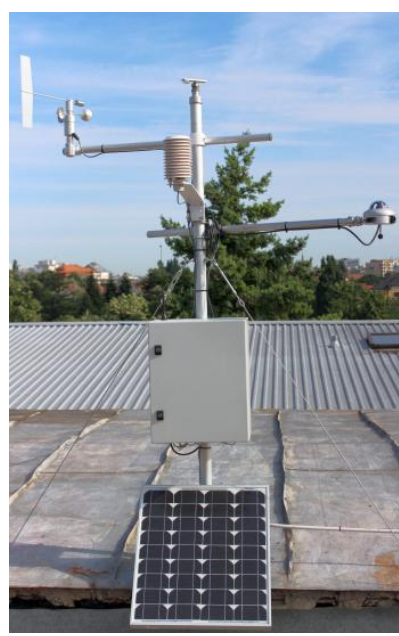

(a)

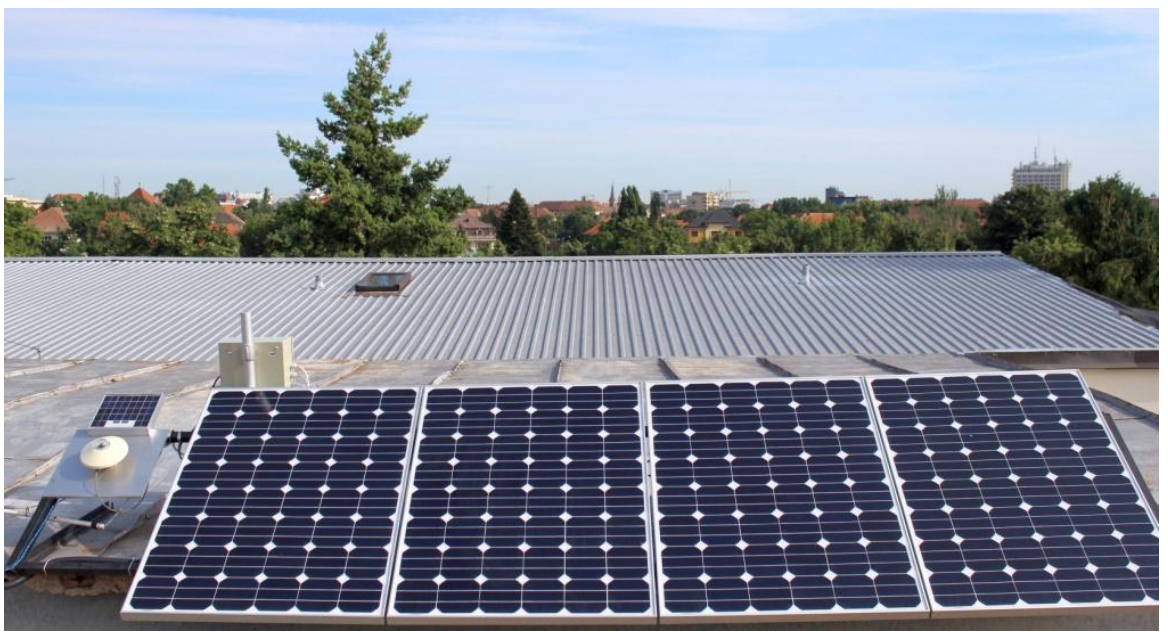

(b)

Fig. 1. (a) A photo of the M-UNIT as part of the FORPV toolkit (b) General view of the experimental PV plant PV-L. The equipment is installed on the Solar Platform of the West University of Timisoara.

\section{Experimental setup}

The toolkit (further identified with the acronym FORPV) was developed within the frame of the Romanian project PN-III-P2-2.1-PED-2016-0592. It consists of two distinctive physical units: (1) The computational unit (C-UNIT) where the forecasting applications are implemented and (2) The PV plant monitoring unit (M-UNIT) which provides radiometric and meteorological data measured in-situ, data required for running the forecasting applications. C-UNIT plays multiple roles, such as real-time control of the forecasting process, database management, and communication with M-UNIT. Figure (1a) shows MUNIT installed on the Solar Platform of the West University of Timisoara. Mainly it consists of an automatic Delta-T Devices [8] meteorological station equipped with the following instruments: (1) a SPN1 pyranometer which measures the global and diffuse (without moving parts) solar irradiances and estimates the sunshine duration (2) standard sensors for air temperature, relative humidity, wind speed and direction; (3) a Delta-T Devices GP2 datalogger and (4) a GPRS modem for communications with C-UNIT. A second experimental setup located also on the Solar Platform was commissioned recently, aiming to 
test the forecast models. This is the experimental PV system of $540 \mathrm{Wp}$ (further identified with the acronym PV-L). A photo of PV-L is presented in Fig. (1b). The PV generator PV-L is connected directly to a constant resistive load of 3.4 Ohms. All the electrical parameters are recorded by the GP2 datalogger. The in-plane solar irradiance is also monitored with a first class pyranometer.

Table1. Sample of data. The columns' meaning is: $R H$ - relative humidity, $T$ - air temperature, $s-$ wind speed, $d$ - wind direction, $G H I$ - global horizontal irradiance, $D H I$ - diffuse horizontal irradiance, SSN - sunshine number [9], $V_{L}$-load voltage , $I_{L}-$ load current

\begin{tabular}{lllllllllll}
\hline Date & Hour & $\begin{array}{l}R H \\
{[\%]}\end{array}$ & $\begin{array}{l}T \\
{\left[{ }^{\circ}\right]}\end{array}$ & $\begin{array}{l}s \\
{[\mathrm{~m} / \mathrm{s}]}\end{array}$ & $\begin{array}{l}d \\
{[\mathrm{deg}]}\end{array}$ & $\begin{array}{l}\text { GHI } \\
{\left[\mathrm{W} / \mathrm{m}^{2}\right]}\end{array}$ & $\begin{array}{l}\text { DHI } \\
{\left[\mathrm{W} / \mathrm{m}^{2}\right]}\end{array}$ & $\begin{array}{l}S S N \\
V_{L}\end{array}$ & $\begin{array}{l}I_{L} \\
{[\mathrm{~V}]}\end{array}$ & {$[\mathrm{A}]$} \\
\hline 11.06 .2018 & $11: 37: 45$ & 56.6 & 27.3 & 0.9 & 138.6 & 308 & 274 & 0 & 17.3 & 5.0 \\
11.06 .2018 & $11: 38: 00$ & 56.7 & 27.3 & 1.1 & 190.7 & 346 & 277 & 0 & 20.4 & 5.9 \\
11.06 .2018 & $11: 38: 15$ & 56.3 & 27.3 & 2.5 & 193.5 & 827 & 261 & 1 & 37.8 & 11.1 \\
11.06 .2018 & $11: 38: 30$ & 56.5 & 27.3 & 3.7 & 154.2 & 817 & 248 & 1 & 38.0 & 11.1 \\
\hline
\end{tabular}

\section{Data and simple models}

The experimental facilities M-UNIT and PV-L are monitored continuously 24/7, all the parameters being measured simultaneously with a sampling rate of 15 seconds. Table 1 illustrates the database structure and the recordings made in a minute, which captures the transition from $S S N=0$ (the Sun is covered) to $S S N=1$ (the Sun is shining). The dimension of the database is relatively large; on each channel the number of the records made in a one hour is 240 while in a one year it is 2102400 .

The SPN1 pyranometer plays a key role in the forecasting process. Although SPN1 is a semiconductor pyranometer, it has many advantages that recommend it in monitoring solar irradiance in the field of a PV plant for forecasting reasons: (1) SPN1 has a response time of the same order as the PV modules. This is a very important characteristic for assessing the PV system performance [10]; (2) it records simultaneously the global and diffuse solar irradiance along with sunshine number; (3) the diffuse component is recorded without a shading ring meaning that it does not require a periodical intervention of a technician. The performance of the SPN1 pyranometer was assessed by comparing the recorded data with the ones measured by the Kipp \& Zonen SMP 10 reference pyranometer [11] (secondary standard according to ISO 9060). Figure 2 compares the measured data ( $G H I$ and $S S N$ ) by the SPN1 and SMP10 in all days of May 2018. Figure (2a) shows the normalized root mean error 
evaluated in every point in respect to the sunshine stability number $(S S S N)$. SSSN is a straightforward indicator of the solar radiative regime stability, counting the changes of SSN in a given period [12]. Figure (2b) shows the matching percentage in evaluating SSN by the two pyranometers. In the sunny days, a very good concordance between the two pyranometers is noticed (for example $P>95 \%$ ). The differences between the two pyranometers increase with increasing the instability of the solar radiative regime.
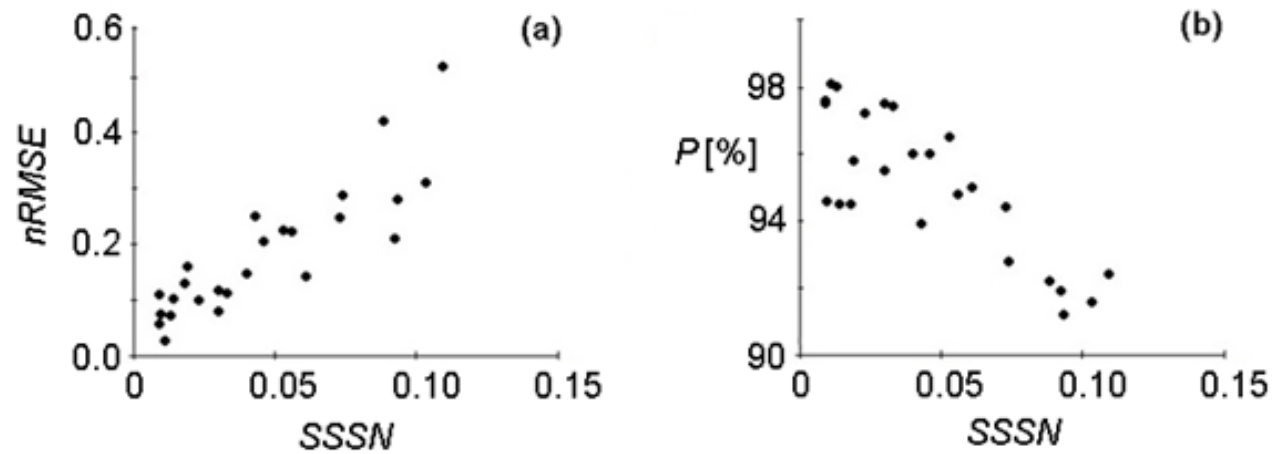

Fig. 2. Comparison between the SPN1 and SMP10 pyranometers to the measurement of: (a) global solar irradiance and (b) sunshine number as function of the daily average of sunshine stability number. $n R M S E$ represents the root mean square error and $P$ is the percentage of sunshine number measurements in which both pyranometers estimate the same value for sunshine number. Each point on the graphs corresponds to a day of May 2018.

An important analysis of the data consists in assessing how the PV-L output power can be correlated with the relevant predictors. An example is given in Figure 3 where the power estimated by linear regression models is compared to the power measured between June 7-20, 2018. The linear regression models consider the main predictors, namely global solar irradiance $G$, diffuse solar irradiance $G_{d}$, air temperature $T$ and sunshine duration $\sigma \equiv \overline{S S N}$ in the given period:

$$
\begin{gathered}
P_{5 m}=65.5-5.3 T+0.631 G-0.151 G_{d}-20.4 S S N \\
P_{1 h}=89.8-6.0 T+0.769 G-0.323 G_{d}-76.6 \sigma
\end{gathered}
$$

$P_{5 m}$ and $P_{1 h}$ represent the instantaneous and the hourly mean power delivered to the load resistor, respectively. Visual inspection of Fig. (3a) shows that $P_{5 m}$ is not linearly correlated with the predictors $\left(R^{2}=94.4 \%\right)$. For this reason, the two-state model was adopted for the online forecasting of the solar irradiance [7]. Figure (3b) shows that $P_{l h}$ is linearly correlated with the main predictors. In this case, a regression analysis of data has led to a very simple linear model for online forecasting of hourly power: 


$$
\hat{P}_{t}\left(P_{t-1}, T, \sigma\right)=351+0.743 P_{t-1}-14.7 T+131 \sigma
$$
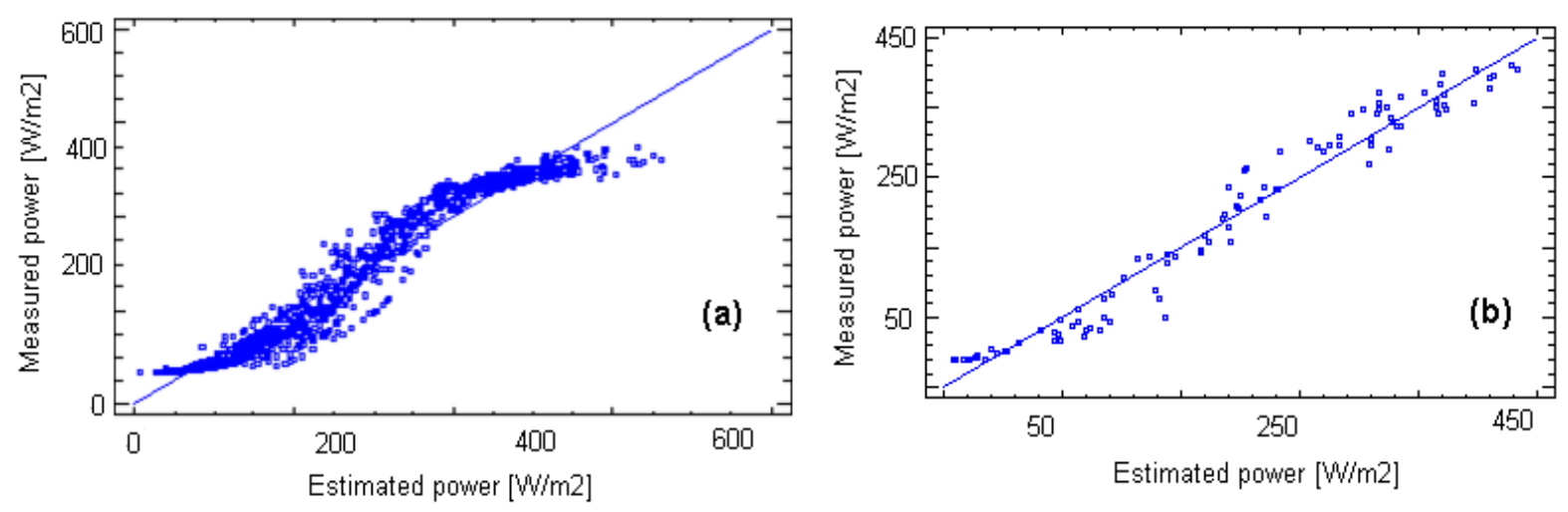

Fig. 3. Measured vs. estimated power delivered by the experimental system PV-L at two intervals of time: (a) instantaneous and (b) hourly mean. The graphs were built with data recorded during 7-20 June 2018.

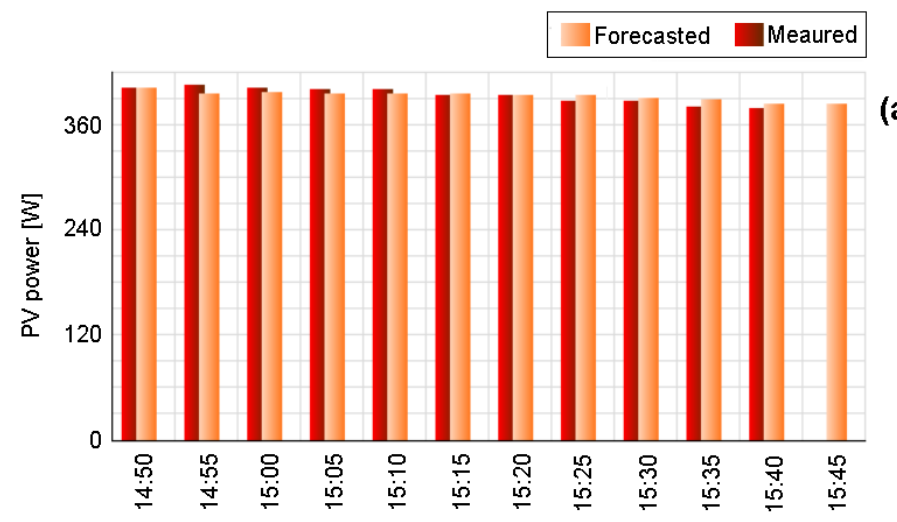

(a)

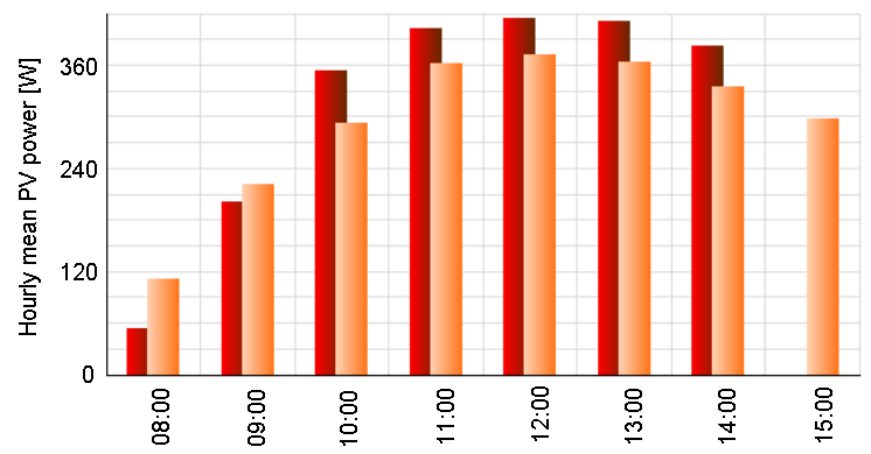

(b)

Fig. 4. Illustration of the PV-L system output power forecasting in August 20, 2018, at two time horizons (a) 5 minutes - instantaneous power is forecasted and (b) one hour - hourly average of the output power is forecasted.

\section{Online forecasting}

The online forecasting procedure is carried out as follows. Using the Microsoft Windows task manager, a 5-minute recurrence event was created. When an event occurs, the 
following operations are performed (sequentially implemented in a Windows batch file): (1) The GP2 datalogger is accessed via the GPRS modem and the data are transferred to the CUNIT database via a parameterized executable file. (2) A MySQL procedure is called which, depending on the current time, generates forecasts for solar irradiance and the output power of the PV-L system at three time horizons: five minutes, one hour and the next day. The web server used is an Apache server from the XAMPP package. All the applications are installed on a computer running Windows 10 operating system.

The forecasts values along with the measurements are available online at http://solar.physics.uvt.ro/forpv/forecasting/ . Figure 4 presents two graphs which compare the forecasted output power of the PV-L system with the measured one at two time horizons. The graphs were captured from screen in August 20 at 15:48. For a 5 minutes forecasting time horizon, the forecast was generated at 15:45, the last vertical bar in Fig. 4a. At 15:50 a bar corresponding to the recorded values is added, and a new forecast is generated. This mechanism runs for every time horizon.

Generally, from Fig. 4 it can be seen that the forecasts trace with reasonable accuracy the measurements. The accuracy decreases with increasing the forecasting time horizon.

\section{Conclusions}

A fully monitored experimental setup for testing the forecasting models for the output power of a PV system was installed in 2018 on the Solar Platform of the West University of Timisoara, Romania (http://solar.physics.uvt.ro/forpv/). The experimental setup includes a combined radiometric/meteorological station and an experimental PV system. As it is designed, the experimental setup constitutes a versatile tool for simultaneously testing of various type of forecasting models at various time horizons. All data are transferred online, and the forecasting results can be accessed also online. The data analysis presented in the paper shows that the autoregressive statistical models (as the two-state model) experienced a reasonable accuracy in nowcasting the solar irradiance. Simple linear regression (with air temperature and relative sunshine as exogeneous predictors) can be used for forecasting hourly mean output power.

\section{Acknowledgements}

This work was supported by a grant of the Romanian National Authority for Scientific Research and Innovation, CNCS/CCCDI - UEFISCDI, project number PN-III-P2-2.1-PED2016-0592. 


\section{References}

[1] T. Tomson, V. Russak, A. Kallis A. Dynamic behavior of solar radiation. In: V. Badescu. (Ed), Modeling Solar Radiation at the Earth Surface. Springer, Berlin, 257-281 (2008).

[2] A. Mills, M. Ahlstrom, M. Brower, A. Ellis, R. George, T. Hoff, B. Kroposki, C. Lenox, N. Miller, M. Milligan, J. Stein, Y-h Wan. IEEE Power Energy M 9 (2011) 33 - 41.

[3] R. Perez, M. David, T.E. Hoff, M. Jamaly, S. Kivalov, J. Kleissl, P. Lauret, M. Perez. Foundations and Trends® in Renewable Energy 1 (2016) 1-44.

[4] J. Kleissl (Ed) Solar energy forecasting and resources assessment. Elsevier, Oxford (2013).

[5] J. Antonanzas, N. Osorio, R. Escobar, R. Urraca, F.J. Martinez-de-Pison, F. AntonanzasTorres. Sol Energy 136 (2016) 78-111.

[6] M. Paulescu, E. Paulescu, P. Gravila, V. Badescu. Springer, London (2013).

[7] M. Paulescu, O. Mares, E. Paulescu, N. Stefu, A. Pacurar, D. Calinoiu, P. Gravila, N. Pop, R. Boata. Energy Convers Manage 79 (2014) 690-697.

[8] Delta-T Devices. WS-GP2 Advanced Automatic Weather Station System https://www.delta-t.co.uk/product/ws-gp2/

[9] V. Badescu, M. Paulescu. Atmos. Res. 101 (2011) 194-204.

[10] M. Paulescu, V. Badescu, C. Dughir. Energy 70 (2014) 49-57.

[11] Kipp \& Zonen. Pyranometers. http://www.kippzonen.com/ProductGroup/3/Pyranometers

[12] M. Paulescu, V. Badescu. Theor. Appl. Climatol. 103 (2011) 459-470. 